\title{
Applications of Strong Interactions between Photons and Molecules to Analytical Sciences
}

\author{
Sunao YAMADA \\ Department of Applied Chemistry, Graduate School of Engineering, Kyushu University, \\ 744 Motooka, Fukuoka 819-0395, Japan
}

\begin{abstract}
Spectroscopic measurements and applications to analytical sciences utilizing strong interactions between photons and molecules will be described. In particular, this report demonstrates high-sensitivity analysis of chemical species in solution through laser multiphoton ionization, and selective detection and characterization of the molecules located at solid surfaces and phase boundaries by using the method of second harmonic generation. Furthermore, new spectroscopic methods and sensitivity improvements utilizing the resonance of incident light photons with surface plasmons emerging on the surfaces of gold nanoparticles and nanostructures are presented. In addition, applications of surface plasmon resonance to spatio-selective plating to microscopic sites and to the field of nanobiotechnology are described, with special focusing on controlling biomolecules.
\end{abstract}

(Received May 15, 2009; Accepted June 26, 2009; Published September 10, 2009)

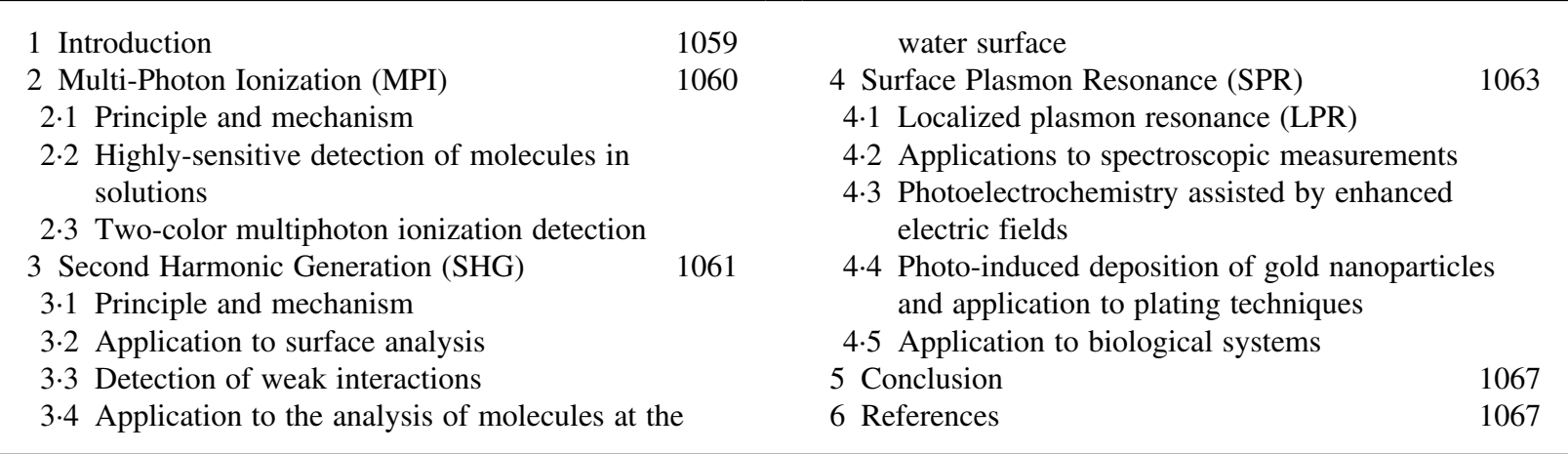

\section{Introduction}

The interaction between light and matter is one of the most important physical phenomena for understanding and developing science and technology. In the field of analytical sciences, the interaction between light and matter functions as a means for transferring energy to the target material and for generating signals which can be used for measuring (detecting) information about the material. For example, in the representative methods of spectrometry, absorptiometry and fluorometry, the measurement (detection) of molecules is accomplished on the basis of their absorption and emission characteristics. These methods for molecular analysis are linear optical processes where a single photon interacts with a single molecule; in other words, they are one-photon processes. However, when using light with high photon density such as laser light, one can induce a nonlinear optical phenomenon where multiple photons interact with a single molecule. Representative phenomena include

E-mail: yamada@mail.cstm.kyushu-u.ac.jp multi-photon ionization and harmonic generation. On the other hand, strong interactions between light and matter can occur even when the intensity of the light is weak, if the phenomenon of surface plasmon resonance (SPR) is used. For example, free electrons on the surfaces of gold and silver nanoparticles oscillate in a collective manner when they are placed inside optical electric fields ranging between the ultraviolet and the near-infrared region. Under the conditions of this SPR phenomenon, drastically amplified electric fields as compared with the electric field of the incident light are locally formed on the surfaces of the nanoparticles. As a result, nonlinear optical processes such as surface-enhanced Raman scattering (SERS) emerge from such interactions between molecules and enhanced electric fields.

Gold and silver nanoparticles themselves also interact with multiple photons, such interactions give rise to various nonlinear processes. In this way, the utilization of strong interactions between photons and molecules can considerably improve the sensitivity of analytical methods based on single-photon processes and can give rise as well to new analytical methods and applications for analytical sciences. In this article, our 
(a)

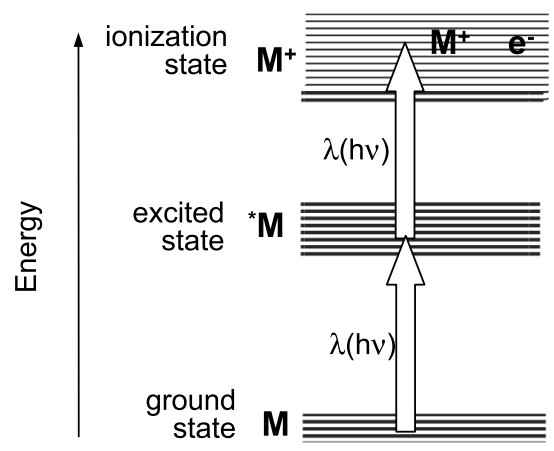

(b)

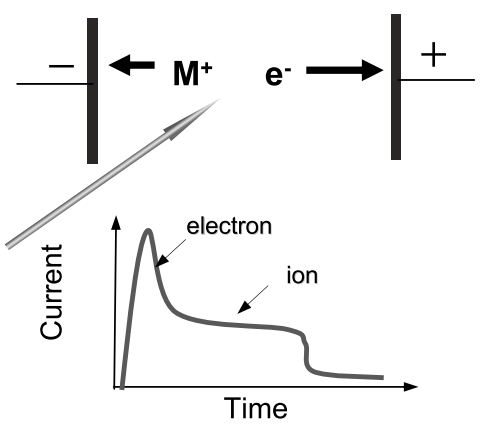

Fig. 1 (a) Basic principle for laser two-photon ionization. (b) Basic concept for detecting photoionization current signals.

research findings for chemical measurements and applications for analytical sciences based on the following specific interactions between photons and molecules are described: (1) multi-photon ionization (MPI), ${ }^{1-3}$ (2) second harmonic generation $(\mathrm{SHG}){ }^{4-7}$ and (3) localized surface plasmon resonance (LSPR). ${ }^{8-11}$

\section{Multi-Photon Ionization (MPI)}

\section{2·1 Principle and mechanism}

Photon fields of the order of $\mathrm{MW} / \mathrm{cm}^{2}$ can be easily generated by using pulsed lasers. Molecules placed in such high photon fields can be excited to energy levels above the ionization threshold, as a result of absorbing multiple photons. Figure 1(a) shows the most representative process of two-photon ionization, where the transition to the excited state is accomplished upon the absorption of one photon, after which the subsequent transition to the ionized state occurs upon the absorption of the second photon. Since the process of one-photon absorption induces the transition to a real excited level, the accurate name for the process is one-photon resonant two-photon ionization.

In any case, when such photoionization is performed inside an external electric field, it is possible to measure the electric current signal originating from the drifts of electrons and of the molecular ions generated in the process of ionization (Fig. 1(b)). The method of resonant ionization presented in Fig. 1(a) allows for larger photoionization currents due to the fact that the ionization yield is considerably higher as compared with non-resonant ionization, which does not resonate with a real excited state. In addition, since resonance ionization occurs via a real excited state, it must be possible to obtain the dependence

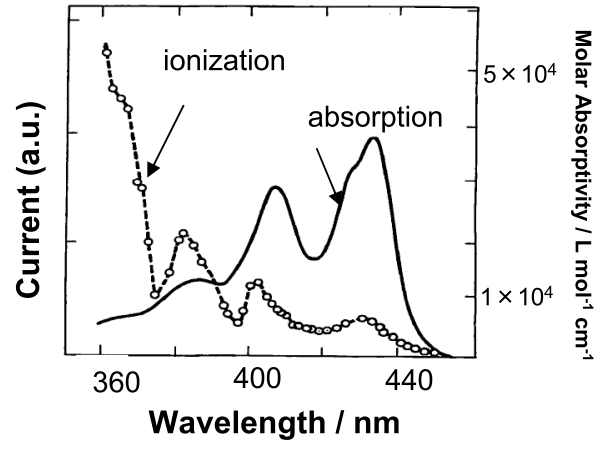

Fig. 2 Two-photon ionization spectrum of perylene $\left(1 \times 10^{-6} \mathrm{M}\right)$ in hexane at a constant photon flux $\left(1 \times 10^{14}\right.$ photons/pulse $)$. The solid line shows the one-photon absorption spectrum.

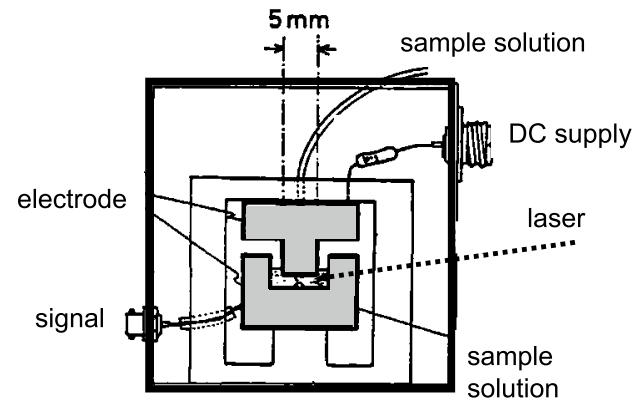

Fig. 3 Schematic diagram of convex-concave type photoionization cell.

of the ionization on the wavelength (in other words, the ionization spectrum) if the ionization is performed by sweeping the wavelength of the laser light. ${ }^{12}$ Figure 2 shows the result for perylene dissolved in hexane. ${ }^{13,14}$ It is clear that there is a good correspondence between the positions of the absorption peaks and those of the ionization peaks. Furthermore, in the case of ionization spectra, there is a tendency of an increase in the signal intensity toward shorter wavelengths (vide infra). Since photoionization is based on the absorption of light, all molecules that can absorb photon(s) are targeted in the measurements. Thus, the range of applicability is wider than that of fluorescence spectroscopy. Also, as the electric current constitutes the signal, it is not affected by the stray light that can cause substantial problems in photodetection methods.

\subsection{Highly-sensitive detection of molecules in solutions}

The author and his colleagues have quickly applied MPI to highly-sensitive detection of molecules in solutions and have published the first report in $1982 .{ }^{15}$ In this case, an important key is the design of ionization cells, and thus the cells were repeatedly improved. A convex-concave flow cell (Fig. 3) proposed in 1983 has been successfully applied in the highly-sensitive detection of pyrene with a concentration of $6 \mathrm{ng} / \mathrm{L} \cdot{ }^{16}$ In the meantime, MPI was also applied for the detector of high-performance liquid chromatography. ${ }^{17-20}$ On the other hand, with the aim of miniaturizing and simplifying the cells, a sub-microliter flow cell with transparent electrodes was developed. ${ }^{21}$ Furthermore, an adjustable photoionization method was proposed by placing the electrodes at the ends of optical fibers. ${ }^{22}$ As can be recognized from these examples, the laser is 
(a)

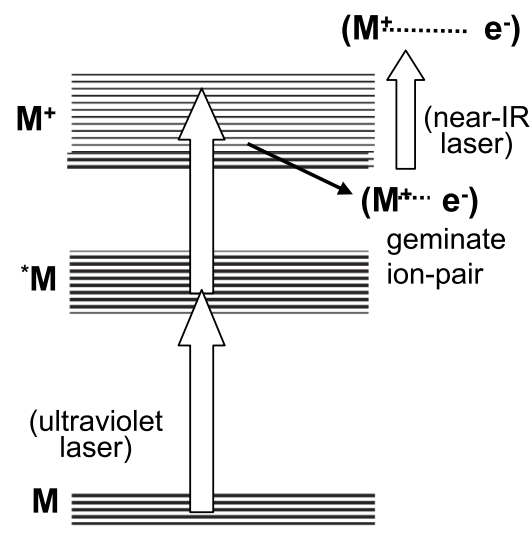

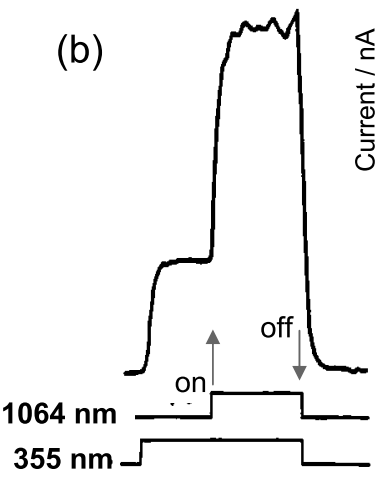

sample solution
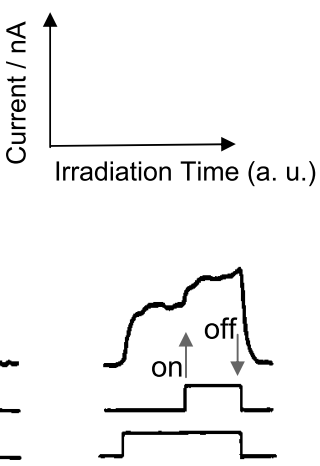

solvent
Fig. 4 (a) Basic principle for two-color multi-photon ionization. (b) Typical photocurrent signals of 9-phenylanthracene (sample solution) and blank signal (solvent) in heptane induced by simultaneous irradiation at 355 and $1064 \mathrm{~nm}$ and by irradiation at $355 \mathrm{~nm}$ alone.

focused in a small region in the MPI method, which makes it advantageous for the detection in extremely confined areas.

\subsection{Two-color multiphoton ionization detection}

As is clear from Fig. 2, a notable difference between the photoionization spectra and the absorption spectra is that the electric current signal in the former becomes relatively larger at shorter wavelengths (vide supra). This implies that the energy difference between the total energy of absorbed light and the ionization threshold (in other words, the excess energy) is related to the strength of the electric current signal. In order to verify this, we have introduced a multi-photon ionization method based on two wavelengths, whose principle is presented in Fig. 4(a). First, thermally equilibrated geminate ion pairs are generated through two-photon ionization. In solution, most of these geminate ion pairs relax into initial molecules, and the probability for dissociation into free ion pairs which can contribute to the electric current is extremely low (several percent). ${ }^{23}$ If these geminate ion pairs are excited further, the distance between the molecular ions and the electrons is increased, and thus the yield of free ion pairs which can contribute to the electric current is expected to increase. From these viewpoints, we have attempted to increase the yield of free ion pairs generated from geminate ion pairs by irradiating the geminate ion pairs with the near-infrared laser light synchronized with the ultraviolet laser light. ${ }^{24,25}$ The results for 9-phenylanthracene dissolved in heptane are presented in

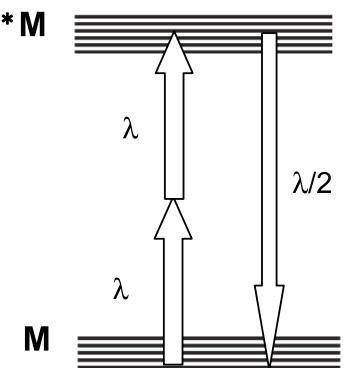

Fig. 5 Basic principle for optical second harmonic generation.

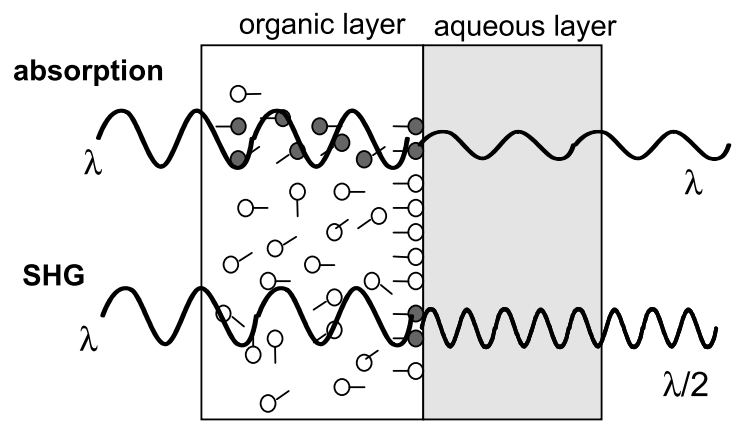

Fig. 6 Comparison of absorption and SHG spectroscopy at liquidliquid phase boundary. In absorption spectroscopy (top) dark-colored molecules are subjected to detection. In SHG spectroscopy (bottom), dark-colored molecules located at the phase boundary are subjected to detection.

Fig. 4(b). When light beams from both the ultraviolet laser $(355 \mathrm{~nm})$ and the near-infrared laser $(1064 \mathrm{~nm})$ are used, the signal becomes up to three times stronger than that in the case where only the ultraviolet laser light is used. On the other hand, in the case of pure solvent, the strength of the signal increased only slightly even when the near-infrared laser was used. As a result, about six-fold increase in the detection sensitivity was achieved. Various kinds of molecules have been detected by this method. ${ }^{26,27}$

\section{Second Harmonic Generation (SHG)}

\subsection{Principle and mechanism}

When an electronically noncentrosymmetric medium is irradiated with light, a phenomenon where two photons with a wavelength $\lambda$ are converted into a single photon with a wavelength $\lambda / 2$ occurs. This phenomenon is known as second harmonic generation (of light), and its principle is illustrated in Fig. 5. Considering this phenomenon at the molecular level, one realizes that the important requisite for the occurrence of SHG is that the electronic states in the molecule must be noncentrosymmetric. In other words, the molecule must hold non-linear hyperpolarizability (more simply, the molecule must have a dipole moment). However, this is not sufficient for observing SHG. The reason for this is explained by considering an example of a two-phase system of liquids (Fig. 6). When SHG-active surfactant molecules are dissolved in an organic phase that contacts with a water phase, the molecules can be asymmetrically aligned at the liquid/liquid phase boundary. We 
(a)

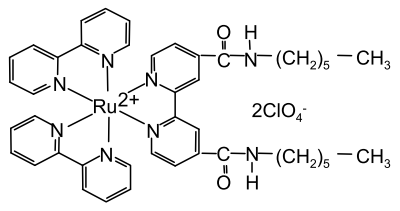

(b)

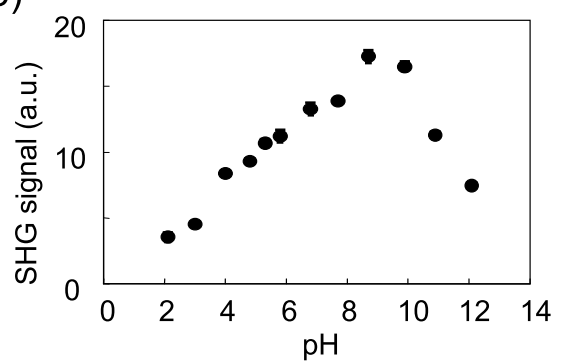

(c)

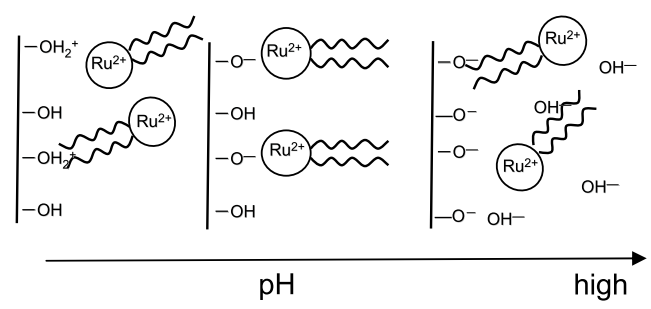

Fig. 7 (a) Molecular structure of Ru-complex. (b) Relationship between SHG signal and $\mathrm{pH}$ of the solution to be measured. (c) Schematic representation for adsorption and desorption of Ru-complex with different $\mathrm{pH}$.

consider the absorption of light with a wavelength $\lambda$ under this condition, as shown in the figure. Although the molecules themselves are SHG-active, the total sum of the dipole moments of the randomly dissolved molecules is zero, and thus SHG is not observed. On the other hand, since the SHG-active molecules at the phase boundary are aligned with noncentrosymmetric orientation, the sum of dipole moments (that is, the non-linear susceptibility) of the molecules does not become zero. Therefore, the SHG signal is collected only from the molecules at the phase boundary, and thus the resultant SHG light can be utilized as a selective measurement method for the molecules at the phase boundaries.

In the measurements of SHG signals, both transmission ${ }^{28}$ and reflection ${ }^{29}$ geometries can be applied, though the optical alignments are somewhat different. In the transmission geometry, a glass plate is immersed into a transparent cell filled with a solution containing SHG-active molecules, and then the laser light $(\lambda)$ irradiates the glass plate under rotation. The SHG signal $(\lambda / 2)$ from the molecules which have adsorbed onto the glass surface is observed. ${ }^{30}$ In the case of the reflection geometry, a prism is brought in contact with the liquid surface, and the SHG signal reflected from the molecules adsorbed onto the prism surface is observed. Since the detection signal in both cases is the laser light, the efficiency of signal collection is high and the influence of stray light is small. The most important characteristic of this method is that it is selective with respect to surfaces and phase boundaries.

\subsection{Application to surface analysis}

Here, we present one example of application to the analysis of the silanol group of a glass plate. ${ }^{28}$ A SHG-active ruthenium (a) Non-linked: SHG-inactive<smiles>O=C([O-])N1c2ccccc2SC2C=CC=CC21</smiles>

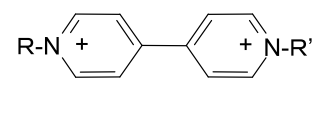

electron donor

electron acceptor

(b) Linked: SHG-active
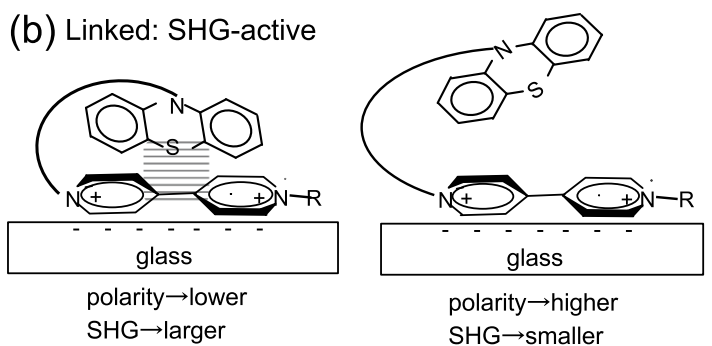

(c)

\begin{tabular}{lcc}
\hline \multicolumn{1}{c}{ Solvent } & $\begin{array}{c}\text { Dielectric } \\
\text { constant }\end{array}$ & $\begin{array}{c}\text { SHG signal } \\
\text { intensity (a.u.) }\end{array}$ \\
\hline $\mathrm{n}$-Hexane & 1.9 & 290 \\
Diethyl ether & 3.1 & 180 \\
Ethyl acetate & 6.2 & 80 \\
\hline
\end{tabular}

Fig. 8 (a) Molecular structures of phenothiazine (left) and viologen (right). (b) Schematic representations of linked molecules adsorbed at the glass surface with different solvent polarities. (c) Relationship between SHG signal and dielectric constant of solvent.

complex (Ru complex: Fig. 7(a)) was dissolved into water and the SHG signals were measured with different $\mathrm{pH}$ values. The results are shown in Fig. 7(b). As can be seen from the figure, the SHG signal quickly increases above $\mathrm{pH} \sim 4$ and starts decreasing above $\mathrm{pH} \sim 8$.

This result can be interpreted as follows. The silanol group at the glass surface is protonated at lower $\mathrm{pH}$, and thus the positively charged head group of the $\mathrm{Ru}$ complex cannot be adsorbed onto the glass surface. As $\mathrm{pH}$ increases, the silanol groups change from an associated state $(-\mathrm{OH})$ to a dissociated one (-O-), and thus the Ru complexes come to be adsorbed onto the glass surface by electrostatic interactions, which induces the appearance of the SHG signal. However, as $\mathrm{pH}$ increases further, the concentration of hydroxide ions in the bulk also increases. Consequently, the $\mathrm{Ru}$ complexes start desorption from the glass surface (Fig. 7(c)), forming ion pairs in the solution, leading to a decrease of the SHG signal. These results suggest that the changes in the SHG signal reflect the dissociation constants of the silanol groups at the glass surface.

\subsection{Detection of weak interactions}

The SHG method can also be applied to the detection of weak interactions between the molecules. ${ }^{31-33}$ For example, phenothiazine and viologen, acting as an electron donor-electron acceptor pair (Fig. 8(a)), are individually SHG-inactive. If a glass plate is immersed into a solution containing a phenothiazineviologen linked compound, the viologen part is adsorbed onto the glass surface and thus the linked molecule is fixed on the glass surface (Fig. 8(b)). When this sample is immersed into a liquid with a different dielectric constant, a SHG signal is observed: the smaller the dielectric constant, the stronger the 
(a)

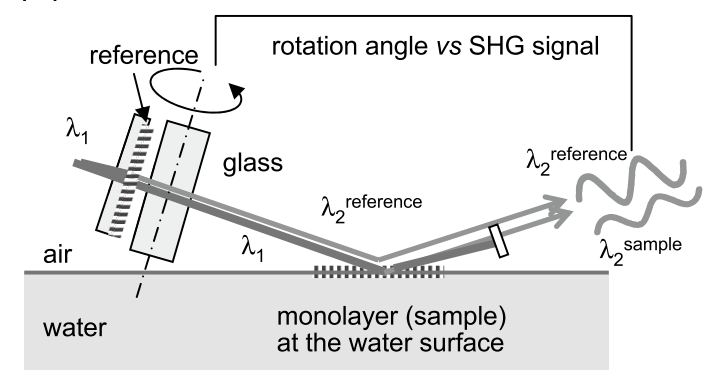

(b)

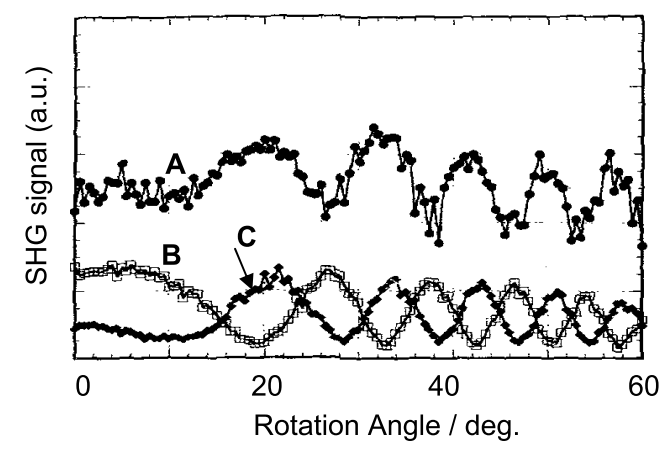

(c)

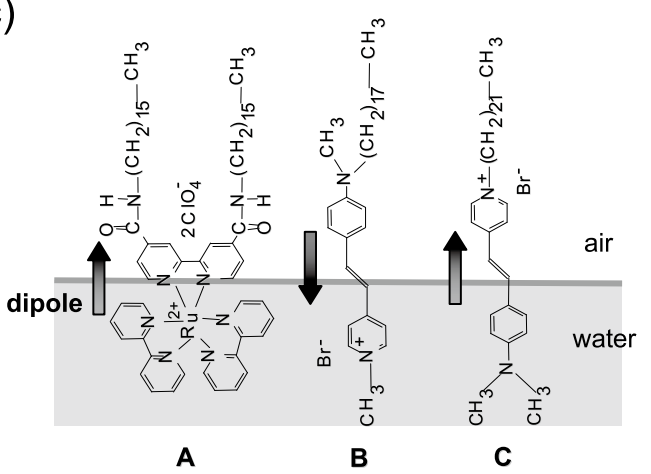

Fig. 9 (a) Basic principle for SHG interference measurements at water surface. (b) SHG interference patterns between the reference material and the sample monolayer (A, B, C: see (c) for molecular structures), as a function of rotation angle. (c) Proposed molecular orientations of dye molecules $\mathbf{A}, \mathbf{B}$, and $\mathbf{C}$, at the water surface.

SHG signal (Fig. 8(c)). ${ }^{31}$ This result can be explained as follows. In a medium with a low dielectric constant, the electrostatic interactions between the phenothiazine and the viologen moieties become prominent, giving the large SHG signal due to interchromophere charge-transfer interaction. Since this type of interaction becomes weaker with increasing the dielectric constant, the SHG signal becomes smaller. Accordingly, the SHG method is found to be powerful for detecting such weak interactions.

\subsection{Application to the analysis of molecules at the water surface}

The SHG method is powerful for the measurements of molecules which are noncentrosymmetrically distributed at the aqueous surfaces. For example, the structure as well as the forming process of mono-molecular films at the aqueous surface can be directly analyzed. Several types of amphiphilic molecules or mixtures of such molecules were dispersed on the aqueous surface in a trough, and the SHG signal was measured consecutively with the fluorescence signal by compressing or extending the aqueous surface, in order to in-situ probe the forming or collapsing processes of mono-molecular films..$^{34,35}$

In the SHG method, the dynamics of mono-molecular films can be detected directly. However, it is impossible to estimate the absolute orientation of the molecules at the aqueous surface. In order to realize such measurements, the author and his colleagues have utilized SHG interferometry, whose principle is presented in Fig. 9(a). ${ }^{36}$ The film of SHG-active molecules prepared on the surface of the glass plate was taken as a reference sample, and the sum of the $\mathrm{SH}$ light emitted from this reference sample and that from the film of SHG-active molecules at the aqueous surface was measured. In this case, if measurements are performed by rotating a glass plate placed between the reference sample and the aqueous surface, an interference pattern of the SH lights emitted from the two sources can be observed. Regarding the mono-molecular films of the SHG-active amphiphilic pigments $\mathbf{A}, \mathbf{B}$ and $\mathbf{C}$, we examined the differences in the orientation profiles of the chromophores (the SHG-active parts) on the basis of the differences between the hydrophobic groups. Such results are presented in Fig. 9(b). It can be seen that the respective phases for pigments $\mathbf{B}$ and $\mathbf{C}$ are inverse, indicating that the chromophores of pigments $\mathbf{B}$ and $\mathbf{C}$ are oriented in opposite directions as to the aqueous surface. ${ }^{37}$ The directions of dipole moments in Ru complex (A) and styrylpyridinium cyanine dyes $(\mathbf{B}, \mathbf{C})$ are shown in Fig. 9(c). The dye $\mathbf{B}$ has a methyl substituent at the pyridinium skeleton, while $\mathbf{C}$ has a long (hydrophobic) alkyl substituent. From the analysis of the interference patterns (Fig. 9(b)), orientations of the three pigments can be illustrated as in Fig. 9(c), where the directions of $\mathbf{B}$ and $\mathbf{C}$ are inverse to each other, in spite of having the same chromophore. Accordingly, by using SHG interferometry, one can perform analysis regarding the absolute orientation of molecules adsorbed at the boundary between air and water. ${ }^{38}$

\section{Surface Plasmon Resonance (SPR)}

\subsection{Localized plasmon resonance (LPR)}

As mentioned at the beginning of this article, there are systems which can induce strong interactions between photons and molecules without the need of using lasers. Typical examples are gold nanoparticles and nanostructures. ${ }^{39}$ The electrons at the surface of gold nanoparticles oscillate in a collective manner, and the phenomenon is referred to as localized surface plasmon. The frequency (wavelength) of this collective oscillation falls in visible the light spectrum in the range between the ultraviolet and the near-infrared region. The condition where the two frequencies match is surface plasmon resonance (SPR), in which the energy of the light is transformed into the energy of the surface plasmon. SPR in closed structures, such as nanoparticles or nanostructures, is called localized surface plasmon resonance (LSPR), which is often distinguished from SPR on open surfaces.

In both cases, under conditions of LSPR (or SPR), electric fields (plasmonic fields) whose strengths are considerably larger than that of the incident light are locally generated in the vicinity of the gold surfaces (Fig. 10(a)). Since the resonance wavelength of the LSPR falls in the region of green light in the case of gold nanoparticles, green light is absorbed, and thus the colloidal solution is red (Fig. 10(b)). Molecules located inside the enhanced electric fields experience non-linear interactions with the electric fields, and induce surface-enhanced Raman scattering 
(a)

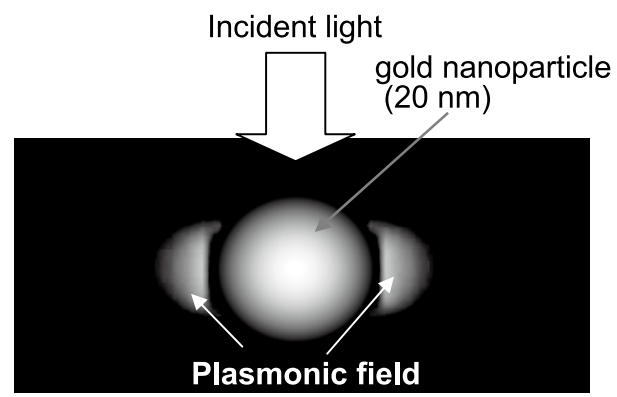

(b)

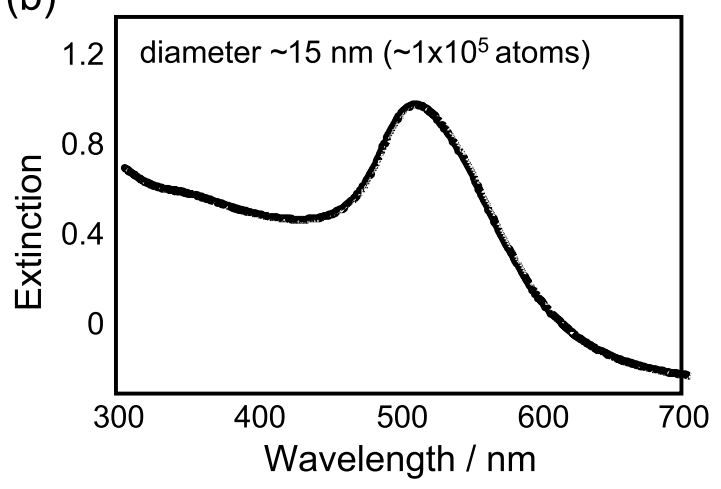

Fig. 10 (a) Image of locally emerged plasmonic fields at the surface of gold nanoparticles. (b) Extinction spectrum of the colloidal solution of gold nanoparticles (15-nm diameter).

(SERS) and changes in the LSPR frequency (wavelength) under the influence of the dielectric constant of the surrounding medium. By focusing on these points, we have been developing tailor-made designs and syntheses of gold nanoparticles and nanostructures, as well as applications to analytical sciences.

\subsection{Applications to spectroscopic measurements}

\section{$4 \cdot 2 \cdot 1$ SERS}

SERS is widely used as a high sensitive method for vibrational spectroscopy. ${ }^{40}$ Although it is well known that silver nanostructures induce a considerable increase in the SERS spectra, we focus on SERS phenomena of gold nanoparticles, since they are much more chemically stable than the silver nanoparticles. In addition, gold nanoparticles are bio-compatible and thus can be superior for wide varieties of analytical applications.

First, we investigated the effect of the size and the agglomeration of spherical gold nanoparticles (nanospheres) on SERS. ${ }^{41,42}$ By comparing the gold nanoparticles with sizes of 35 and $15 \mathrm{~nm}$, researchers found that the former produces a larger SERS signal. Also, in the agglomerated structures formed through sedimentation, it was found that partially fused structures produce a larger SERS signal than the isolated nanoparticles.

Gold nanoparticles with different shapes have also been compared. ${ }^{43}$ The films of gold monoparticles, nanospheres and nanorods (rod-shaped gold nanoparticles) ${ }^{44}$ were fabricated at a liquid/liquid phase boundary, and they were scooped by using a glass plate to use as SERS substrates. SEM images of the nanoparticle films are shown in the right column of Fig. 11. The long axes of the two nanorods are almost the same, but the short axes are different. All films have well-packed nanoparticle-level domains. Raman scattering spectra of

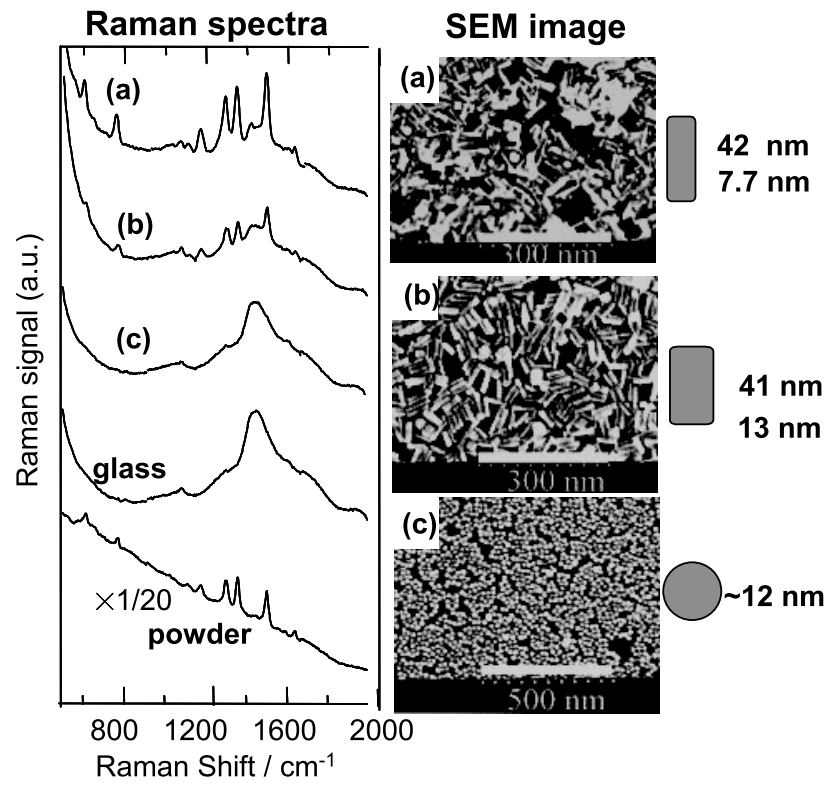

Fig. 11 Non-resonance SERS spectra of Rhodamine 6G on the gold nanorods with high aspect ratio (a: length, $42 \mathrm{~nm}$; diameter, $7.7 \mathrm{~nm}$ ) and low aspect ratio (b: length, $41 \mathrm{~nm}$; diameter, $13 \mathrm{~nm}$ ), and on gold nanosphere (c: diameter, $12 \mathrm{~nm}$ ). Together, the spectra at the glass surface (glass) and the powder sample (powder) are shown.

Rhodamine 6G (R6G) are shown in the left column of Fig. 11, together with the spectrum of R6G powder having characteristic peaks at 1311, 1363, and $1508 \mathrm{~cm}^{-1}$ (powder, in the left column of Fig. 11). It is clear that the signal intensity of the nanorod with large aspect ratio (Fig. 11(a)) is considerably larger than that with small aspect ratio (Fig. 11(b)). On the other hand, no appreciable peaks due to R6G are detected from the nanosphere (Fig. 11(c)); the broad band around $1450 \mathrm{~cm}^{-1}$ is ascribed to the scattering from the glass plate (glass, in the left column of Fig. 11). Although gold nanorods have been used as SERS substrates, none of those studies have explored the differences associated with the aspect ratio as examined by the authors. If the aspect ratio and the alignment pattern (orientation and density) can be fully controlled, it is expected that more quantitative and useful knowledge can be obtained. These aspects provide very important implications for the fabrication of superior SERS substrates.

\subsubsection{SPR on gold nanorods}

A representative transmission electron micrograph (TEM) image of gold nanorods is shown in Fig. 12(a), together with the absorption spectrum of the colloidal solution. The gold nanorods show two surface plasmon (SP) bands as a result of their shape anisotropy, where the SP band along the short axis shows a peak in the visible region $(\sim 520 \mathrm{~nm})$, while that along the long axis shows a strong peak in the far-red to near-infrared region. In particular, the latter peak shifts considerably with the aspect ratio and with the dielectric constant of the surrounding medium. ${ }^{45}$

Although we omit here the methods for synthesizing gold nanorods, they can be stabilized with a cationic surfactant, cetyltrimethylammonium bromide (CTAB). We have successfully fixed gold nanorods onto the surface of a glass plate by utilizing electrostatic interactions. ${ }^{46,47}$ More specifically, after sequentially adsorbing a polycation poly(allyamine 
(a)
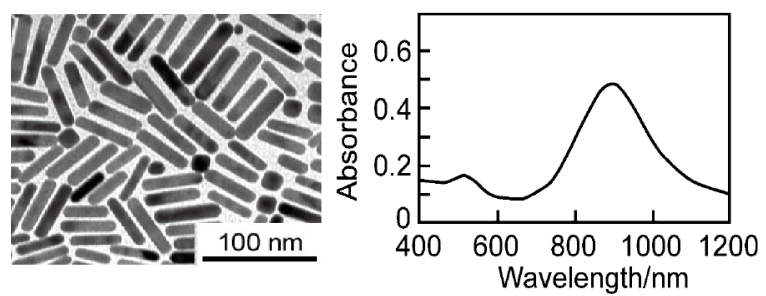

(b)

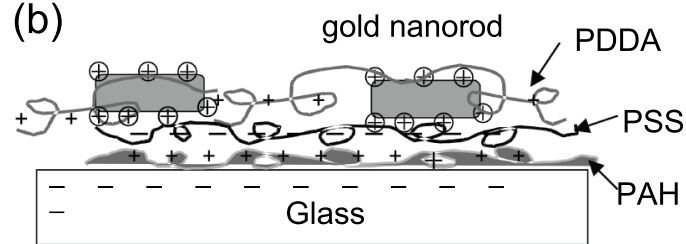

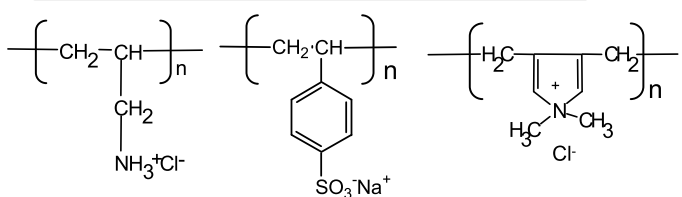

PAH

PSS (c)

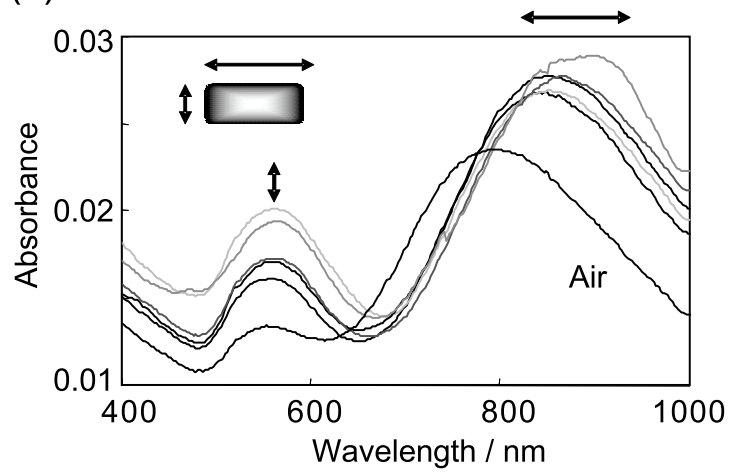

Fig. 12 (a) TEM image (left) and absorption spectrum (right) of the colloidal solution of gold nanorods. (b) Estimated structure of gold nanorods-polyion assembly on the glass surface. (c) Absorption spectra of the nanorod-modified glass plate in air (air) and in solvents (water, ethanol, acetone, cyclohexane, and chloroform, in the order of red-shift).

hydrochloride) (PAH) and a polyanion poly(sodiumstyrene sulfonate) (PSS) onto a glass plate, the plate was immersed into the aqueous colloidal solution of gold nanorods, which adsorbed onto the plate by electrostatic interactions. Then, the plate was immersed into a polycation, poly(diallyldimethyl ammonium chloride) (PDDA), in order to confine the nanorods in the polymer layer, as is illustrated in Fig. 12(b). The absorption spectrum obtained when the prepared plate was immersed into a medium with a different refractive index is shown in Fig. 12(c). In contrast to the SP band of the short axis, which is virtually independent of the medium, the SP band of the long axis shifts considerably depending on the medium. For example, when a medium with a diffractive index of 1.33 (water) is replaced with a medium whose index is 1.45 (chloroform), the peak shifts by $\sim 35 \mathrm{~nm}$. Accordingly, it is possible to estimate the refractive index by using the peak shift of the SP band of the long axis. Therefore, the SPR sensing based on transmission geometry can be realized by using the gold nanorods. (a)

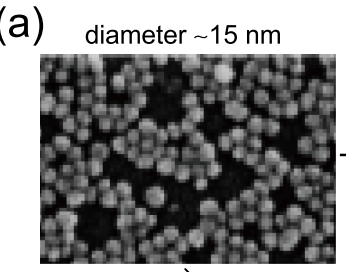

(c)

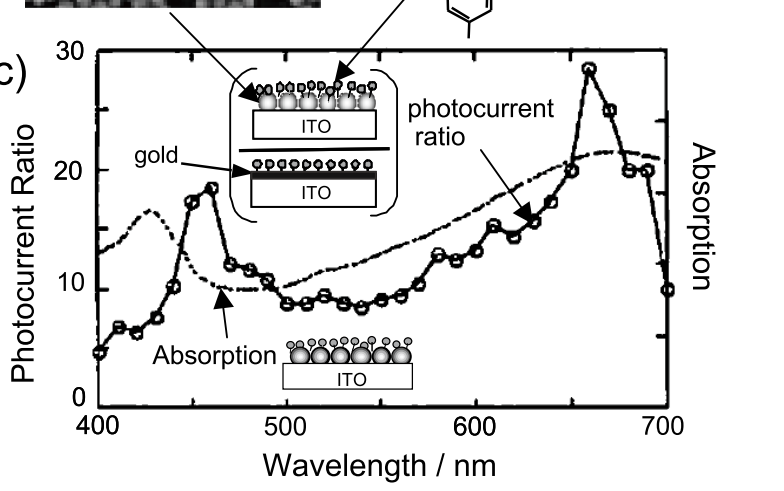

Fig. 13 (a) SEM image of gold nanoparticle film. (b) Molecular structure of a porphyrin derivative. (c) Photocurrent ratios for gold nanoparticle film versus planar gold film $(\bigcirc)$ and absorption spectrum of gold nanoparticle film (-).

\subsection{Photoelectrochemistry assisted by enhanced electric fields}

Under the condition that electrical conductivity is retained, gold nanoparticle multistructures can be used as threedimensional porous electrodes. ${ }^{48-50}$ They are expected to have the following effects: increase in the electrode surface area and increase in molecular excitation efficiency through the locally enhanced electric fields. Regarding the latter effect, reports have been limited to the focusing on flat electrodes, and not yet to three-dimensional nanostructures. In order to elucidate this issue, we prepared a monoparticle film of gold nanoparticles by utilizing a liquid/liquid phase boundary. ${ }^{43}$ This film was deposited onto a transparent electrode and was used as a three-dimensional electrode. An scanning electron micrograph (SEM) image of the electrode is shown in Fig. 13(a). Next, a porphyrin pigment (Fig. 13(b)) was attached to the gold surface by self-assembly, and the photocurrent was measured. For comparison, we also measured the photocurrent from the corresponding flat gold electrode. The ratio of the photocurrents, three-dimensional $v s$. flat electrodes, was taken as a function of wavelength, as shown in Fig. 13(c). If the ratio depends only on the amount of immobilized pigment, it must be constant irrespective of the wavelength. However, the ratio of the photocurrents exhibits a clear dependence on the wavelength and corresponds well to the transmission absorption spectrum of the three-dimensional electrode. ${ }^{51}$ Therefore, when gold nanoparticles are used, the photocurrent signal increases in the regions where the surface plasmons are excited, which results in the improvement of the photoelectrochemical sensitivity. ${ }^{52}$

\subsection{Photo-induced deposition of gold nanoparticles and application to plating techniques}

When a colloidal cyclohexane solution of dodecanethiol (DT) capped gold nanoparticles are irradiated with a pulsed laser light, giant particles of several-hundred $\mathrm{nm}$ sizes are generated..$^{53}$ At the same time, deposition (fixation) of gold nanoparticles on the solid surface was found to proceed. ${ }^{54-58}$ Taking advantages 
(a)

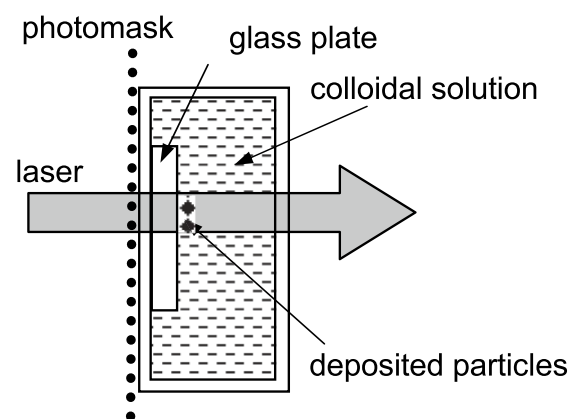

(b)

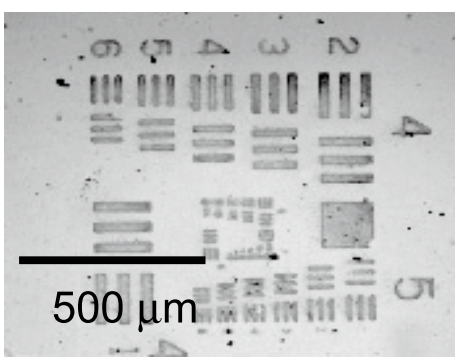

(c)

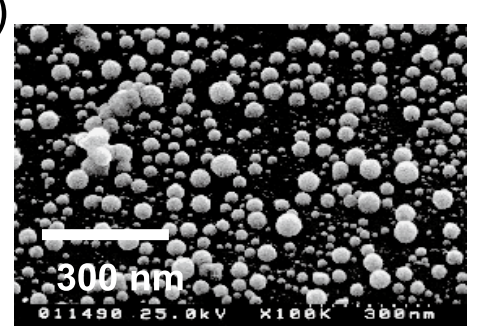

Fig. 14 (a) Experimental geometry for laser-induced deposition. (b) Macroscopic and (c) SEM images of the deposited gold nanoparticles on the glass surface.

of this deposition phenomenon, we can propose a new plating technique. The basic principle is shown in Fig. 14(a). First, a glass plate is immersed into the above colloidal solution and a 532-nm pulsed laser light irradiates it through a photomask. Then, the nanoparticles are fixed onto the glass plate following the pattern of the photomask (Fig. 14(b)). The deposition of gold nanoparticles is confirmed from the observation of SEM images (Fig. 14(c)).

We focused on the potential use of gold nanoparticles as a catalyst for electroless plating, and attempted to perform electroless copper plating by using the deposited gold nanoparticles. ${ }^{59}$ Figure 15(a) shows an optical image of the copper plating on the inner wall of a glass capillary (outer diameter $0.5 \mathrm{~mm}$ ). First, the above-mentioned gold colloidal solution is injected into the capillary through capillary action, and then a pulsed laser is irradiated. After removal of the colloidal solution, a solution for copper plating is injected and copper plating is performed. In the same manner, copper plating can be applied to a spatioselective part of a non-conductive membrane filter, in order to make this part conductive (Fig. 15(b)).

In the case of fixating the catalyst onto the inside of the flow paths in microreactors, or implementing the wiring, it is necessary to perform those operations before preparing the flow paths. However, if this method is applied, such an operation may be performed even after construction of the flow paths. Thus, the present method can be expected to find applications in (a)

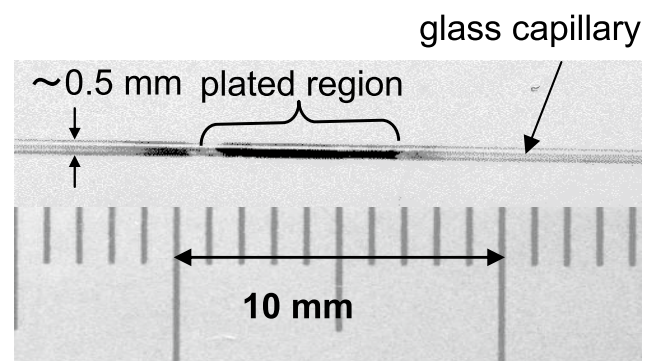

(b)
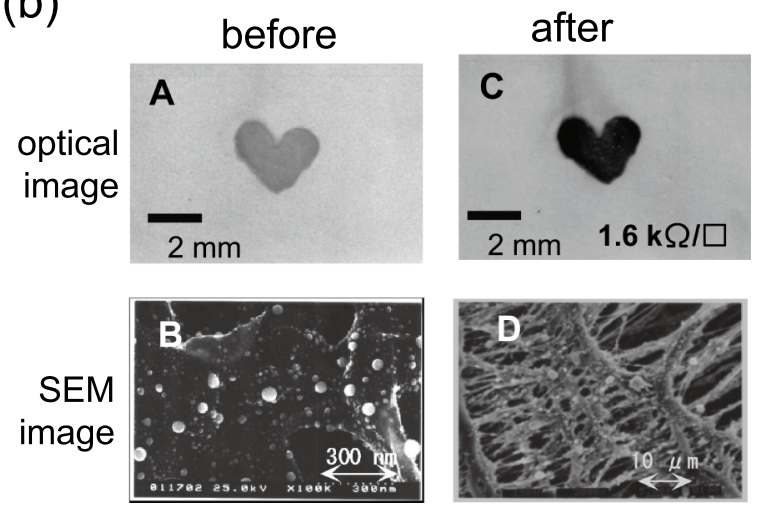

Fig. 15 (a) Macroscopic photograph of the glass tube after electroless copper plating of the gold-nanoparticle-deposited region. (b) Macroscopic (A, C) and SEM (B, D) images of PTFE membrane filters before $(\mathrm{A}, \mathrm{B})$ and after $(\mathrm{C}, \mathrm{D})$ electroless copper plating of gold-nanoparticle-deposited region.

the field of chemical microsystems. Also, this method has been successfully applied to the fabrication of substrates for Raman and infrared spectroscopy. ${ }^{60,61}$

\subsection{Application to biological systems}

When photochemical (photophysical) treatments are performed in tissues or organs, the wavelength of the incident light frequently becomes important. Especially, if one needs the irradiation light to penetrate into the tissues, the light of the near-infrared region is superior to the visible or ultraviolet light because of higher transmissivity to the tissues. Since the gold nanorod has the strong plasmon (absorption) hand in the far-red to near-infrared region, it can be superior to the gold nanosphere when the photochemical (photophysical) treatments or in-situ probing of the inner part (e.g., blood vessels) of the tissues are carried out using gold nanoparticles. From these viewpoints, we have been trying to apply the gold nanorods to biological systems.

As mentioned above, gold nanorods are shielded with a cationic surfactant CTAB. However, CTAB is toxic to living organisms, and therefore its use in the biological applications is not desirable. From this viewpoint, we have succeeded in preparing the gold nanorods where some of the CTAB molecules were replaced with a phospholipid, phosphatidylcholine (PC). ${ }^{62}$ Consequently, the resulting nanorods were found to be biocompatible, since they induced no substantial damage to HeLa cells.

As an example of utilizing the above-described biocompatible gold nanorods, we have tried photoinduced release of DNA using the near-infrared light and the gold nanorods. A conceptual 
(a)

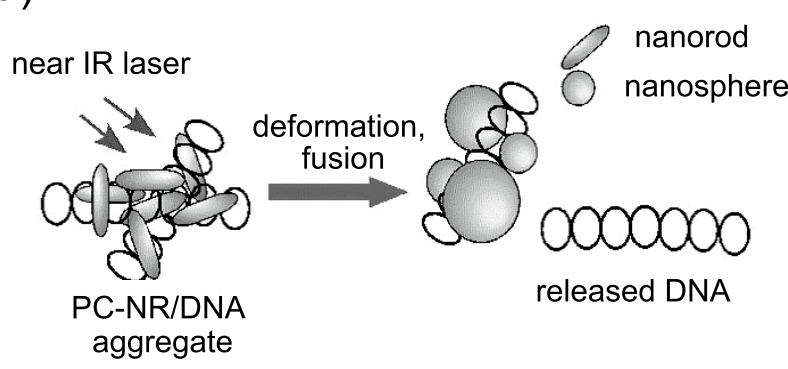

(b)

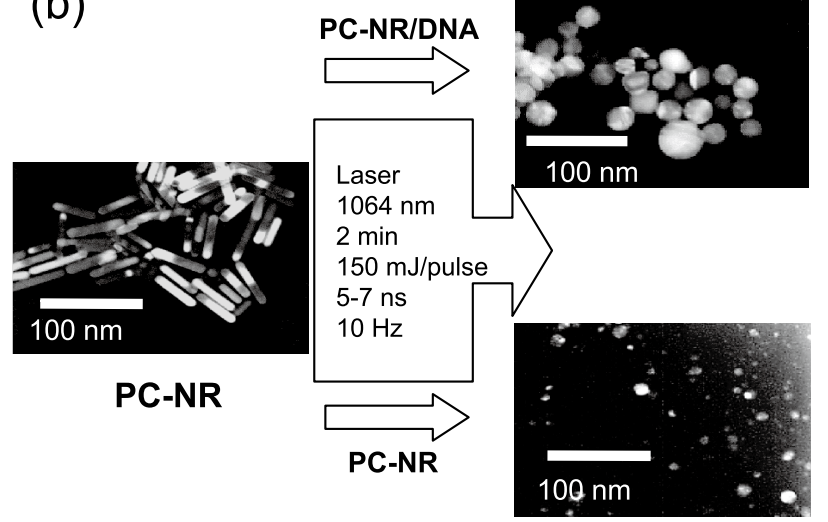

Fig. 16 (a) Basic principle of laser-induced release of DNA using gold nanorods. (b) TEM images of the aggregates of phosphatidylcholine-modified gold nanorods (PC-NR) and DNA (PC-NR/DNA): left, PC-NR; right top, after laser irradiation of PC-NR/DNA; right bottom, laser irradiation of PC-NR.

diagram of the process is shown in Fig. 16(a). ${ }^{63,64}$

Although some of the CTAB could be replaced with PC, the resultant nanorods are still cationic. On the other hand, DNA exhibits anionic properties. Thus, the electrostatic interaction between plasmid DNA and the above-described gold nanorods can generate aggregates. Then, when the aggregates are irradiated with 1064-nm laser light, the free electrons in the gold nanorods become excited. Since the subsequent relaxation process is exothermic, the nanorods are heated. As a result, the gold nanorods cause shape changes into spheres. Nevertheless, they are mostly trapped with DNAs, and thus they cause fusion and form large spherical particles. This then reduces the number of particles which can interact with DNAs, so that some of the DNAs are released from the aggregates. In fact, the process is essentially valid, since the irradiation of aggregates creates large spherical particles, as can be seen from the TEM image (right top of Fig. 16(b)). When only the gold nanorods were irradiated, fragmentation occurred preferentially, resulting in smaller spherical particles, as could be recognized from the TEM image (right bottom of Fig. 16(b)). By comparing the results of electrophoresis before and after irradiation of the laser light, one can confirmed that some DNAs are certainly released from the aggregate. The results indicate that the photoinduced release of DNA is possible by using near-infrared light. Although the conditions for the actual application of the method are still not optimized, we can propose a new method for light-driven drug delivery by taking the most of surface plasmon resonance.

\section{Conclusion}

This article assembled research results on spectroscopic measurements and applications to analytical sciences based on the strong interactions between photons and molecules. The fields which induce such strong interactions are called "strong photon-molecule coupling fields", and they are attracting much attention, especially in the field of plasmonics. In such strong electric fields, the molecules experience non linear interactions with electromagnetic fields, and thus the resultant signals to be detected increase exponentially on the intensity of light. In fact, in the methods of two-photon ionization and SHG, the signal depends on the square of the intensity of the incident light. This indicates that the precise design and implementation of the coupling fields is basic and extremely important, and this is also valid in the case of LSPR for gold nanoparticles and nanostructures. In the SERS, LSPR, and photoelectrochemical methods described here, it is crucial to establish the methods for the design and reproducible fabrication of tailor-made gold nanoparticles and nanostructures, in order to achieve high-performance detection and determination of molecules.

As to spectroscopy, improvement of reliability is even more important than improvement of the sensitivity. Since one of the advantages on the strong interactions between photons and molecules is its extremely high sensitivity, efforts in the future should be directed towards improving the reliability of the method.

\section{References}

1. S. Yamada, Chem. Chem. Ind., 1987, 40, 120.

2. S. Yamada, Bunseki, 1989, 7, 575.

3. S. Yamada, Bunseki, 1994, 4, 272.

4. S. Yamada, Bunseki, 1996, 9, 721.

5. S. Yamada and I. S. Lee, Anal. Sci., 1998, 14, 1045.

6. S. Yamada, Bunseki, 2000, 3, 148.

7. S. Yamada, Bunseki, 2006, 11, 550 .

8. S. Yamada, O plus E, 2006, 28, 503.

9. S. Yamada, Electrochemistry, 2006, 74, 496.

10. S. Yamada and T. Akiyama, in "Recent Advances on Design and Applications of Plasmonic Nanomaterials", ed. S. Yamada, 2006, CMC, Tokyo, 63.

11. I. Uechi and S. Yamada, Anal. Bioanal. Chem., 2008, 391, 2411.

12. N. Sato, S. Yamada, and T. Ogawa, Anal. Sci., 1987, 3, 109.

13. S. Yamada, N. Sato, H. Kawazumi, and T. Ogawa, Anal. Chem., 1987, 59, 2719.

14. S. Yamada, Bunko Kenkyu, 1989, 38, 259.

15. S. Yamada, K. Kano, and T. Ogawa, Bunseki Kagaku, 1982, 31, E247.

16. S. Yamada, A. Hino, K. Kano, and T. Ogawa, Anal. Chem., 1983, 55, 1914.

17. S. Yamada, A. Hino, and T. Ogawa, Anal. Chim. Acta, 1984, 156, 273.

18. S. Yamada, A. Hino, and T. Ogawa, Bunseki Kagaku, 1984, 33, E37.

19. S. Yamada, C. Sakane, and T. Ogawa, Talanta, 1987, 34, 461.

20. S. Yamada, T. Ogawa, and P. Zhang, Anal. Chim. Acta, 1986, 183, 251.

21. S. Yamada, Anal. Chem., 1991, 63, 1894.

22. S. Yamada, Anal. Chim. Acta, 1992, 264, 1. 
23. B. S. Yakovlev and L. V. Lukin, "Photoionization in Non-polar Liguids", ed. K. P. Lawley, 1985, John Wiley and Sons, New York, 99.

24. S. Yamada, Anal. Chem., 1988, 60, 1975.

25. S. Yamada, Anal. Chem., 1989, 61, 612.

26. S. Yamada, Spectrosc. Lett., 1989, 22, 405.

27. S. Yamada and I. Shinno, Talanta, 1989, 36, 937.

28. Y. Niidome, S. Tagawa, and S. Yamada, Colloids Surf., A, 2002, 198, 467.

29. Y. Yamada, T. Nakano, K. Yamada, T. Matsuo, and S. Yamada, Colloids Surf., A, 2000, 163, 233.

30. S. Yamada, Y. Yamada, T. Nakano, and T. Matsuo, Chem. Lett., 1994, 23, 937.

31. A. Toki, H. Yonemura, S. Yamada, and T. Matsuo, Chem. Lett., 1995, 24, 741.

32. K. Yamada, H. Otsubo, H. Yonemura, S. Yamada, and T. Matsuo, Chem. Lett., 1997, 26, 451.

33. K. Yamada and S. Yamada, J. Colloid Interface Sci., 1999. $216,440$.

34. T. Nakano, Y. Yamada, T. Matsuo, and S. Yamada, $J$. Phys. Chem. B, 1998, 102, 8569.

35. K. Yamada, H. Yonemura, T. Matsuo, and S. Yamada, $J$. Photochem. Photobiol., A, 1998, 114, 163

36. S. Yamada, H. Iida, and T. Matsuo, Ber. Bunsen-Ges. Phys Chem., 1997, 101, 672.

37. T. Nakano and S. Yamada, Bunseki Kagaku, 1998, 47, 979.

38. S. Yamada and T. Nakano, Anal. Sci., 2000, 16, 1233.

39. S. Yamada, "Recent Advances on Design and Applications of Plasmonic Nanomaterials", 2006, CMC, Tokyo.

40. R. K. Chang and T. E. Furtak (ed.), "Surface Enhanced Raman Scattering", 1982, Plenum, New York

41. M. Suzuki, Y. Niidome, Y. Kuwahara, N. Terasaki, K. Inoue, and S. Yamada, J. Phys. Chem. B, 2004, 108, 11660.

42. M. Suzuki, Y. Niidome, and S. Yamada, Colloids Surf., A, 2006, 284, 388.

43. M. Suzuki, Y. Niidome, N. Terasaki, K. Inoue, Y. Kuwahara, and S. Yamada, Jpn. J. Appl. Phys., 2004, 43 L554.

44. S. Yamada, "Hyomen (in Japanese)", 2004, 42, 10.
45. S. Link and M. A. El-Sayed, J. Phys. Chem. B, 1999, 103 , 8410.

46. Y. Niidome, H. Takahashi, S. Urakawa, K. Nishioka, and S. Yamada, Chem. Lett., 2004, 33, 4, 454.

47. N. Omura, I. Uechi, and S. Yamada, Anal. Sci., 2009, 25 , 255.

48. Y. Kuwahara, T. Akiyama, and S. Yamada, Langmuir, 2001, 17, 5714.

49. T. Akiyama, K. Inoue, Y. Kuwahara, N. Terasaki, Y. Niidome, and S. Yamada, J. Electroanal. Chem., 2003 550,303

50. T. Akiyama, K. Inoue, Y. Kuwahara, Y. Niidome, N Terasaki, S. Nitahara, and S. Yamada, Langmuir, 2005, 21, 793

51. T. Akiyama, M. Nakada, N. Terasaki, and S. Yamada, Chem. Commun., 2006, 395

52. N. Terasaki, S. Nitahara, T. Akiyama, and S. Yamada, Jpn. J. Appl. Phys., 2005, 44, 2795.

53. Y. Niidome, A. Hori, T. Sato, and S. Yamada, Chem. Lett., 2000, 310 .

54. Y. Niidome, A. Hori, H. Takahashi, Y. Goto, and S. Yamada, Nano Lett., 2001, 1, 365.

55. H. Takahashi, Y. Niidome, T. Sato, and S. Yamada, Colloids Surf., A, 2004, 247, 105.

56. H. Takahashi, Y. Niidome, and S. Yamada, Bunseki Kagaku, 2002, 51, 797 .

57. K. Honda, Y. Niidome, H. Kawazumi, and S. Yamada, Bunseki Kagaku, 2006, 55, 675.

58. Y. Niidome, H. Takahashi, T. Kawasawa, A. Hori, and S. Yamada, Jpn. J. Appl. Phys., 2003, 42, 7640.

59. S. Yamada, Bunseki Kagaku, 2008, 57, 801 .

60. Y. Niidome, H. Hisanabe, A. Hori, and S. Yamada, Anal. Sci., 2002, 17, i1185.

61. H. Hisanabe, Y. Niidome, H. Takahashi, and S. Yamada, Bunseki Kagaku, 2003, 52, 661.

62. H. Takahashi, Y. Niidome, T. Niidome, K. Kaneko, H Kawasaki, and S. Yamada, Langmuir, 2006, 22, 2.

63. H. Takahashi, Y. Niidome, and S. Yamada, Chem Commun., 2005, 2247.

64. H. Takahashi, T. Niidome, A. Nariai, Y. Niidome, and S Yamada, Chem. Lett., 2006, 35, 500. 